\title{
Ability of Calcium Silicate and Epoxy Resin-based Sealers to Fill the Artificial Lateral Canals in the Presence or Absence of Smear Layer
}

Amin SALEM MILANI ${ }^{a}$, Sepideh KUZEGARI ${ }^{a}$, Vahid ZANDa, Hadi MOKHTARI ${ }^{a}$, Mahdi RAHBAR ${ }^{\mathrm{b}}$

aEndodontic Department, Faculty of Dentistry, Tabriz University of Medical Sciences, Tabriz, Iran

bsthetic and Restorative Dentistry Department, School of Dentistry, Ardabil University of Medical Sciences, Ardabil, Iran

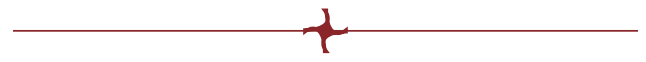

\section{ABSTRACT}

Background: The lateral canals are pathways for bacteria and their products to pass between the root canal and the periradicular tissue.

Objectives: The present study aims to compare the filling ability of the lateral canals with three calcium silicate based sealers, including MTA Fillapex, Endoseal MTA and Sure-Seal Root, and AH26 epoxy resin sealer in the presence and absence of the smear layer.

Material and methods: Six lateral canals were prepared using an engine reamer in 80 single-rooted human teeth. The root canals were cleaned, then the teeth were randomly divided into two groups. In group $A$, the smearlayer was removed using $17 \%$ EDTA and $5.25 \% \mathrm{NaOCl}$, and in group $\mathrm{B}$, the canals were irrigated with normal saline. Groups $A$ and $B$ were divided into four subgroups each, according to the sealer used: $A 1, B 1$ (MTA Fillapex sealer), A2, B2 (Endoseal MTA sealer), A3, B3 (Sure-Seal Root sealer), and A4, B4 (AH26 sealer). Obturation of canals was conducted by the warm vertical technique and then teeth were incubated for 72 hours. Teeth were made clear and the filling of lateral canals were evaluated under stereomicroscope.

Results: In group A, the highest lateral canals filling rate was in subgroup A2 and the lowest in subgroup A1, and the difference was statistically significant. However, in both groups, the highest lateral canals filling rate

\footnotetext{
Address for correspondence:

Sepideh Kuzegari

Mailing address: Endodontic Department, Faculty of Dentistry, Tabriz University of Medical Sciences, Tabriz, Iran

Tel.: +989141291285

Email:s_tajhizat@yahoo.com

and

Mahdi Rahbar

Mailing address: Esthetic and Restorative Dentistry Department, School of Dentistry, Ardabil University of Medical Sciences, Ardabil, Iran Tel.: +989141543176

Email: mahdirhbr@gmail.com
}

Article received on the $5^{\text {th }}$ of July 2021 and accepted for publication on the $29^{\text {th }}$ of September 2021 
was in subgroups B2 and B3 and the lowest in subgroup B1. However, the difference was not statistically significant. In addition, no significant difference was observed in the lateral canals filling rate considering their position in the root.

Conclusion: Providing the removal of the smear layer, calcium silicate-based sealers, Endoseal MTA and Sure-Seal Root had a higher ability to fill the lateral canals than AH26 and MTA fillapex sealers.

Keywords: : calcium silicate-based sealer, epoxy resin-based sealer, lateral canals, smear layer.

\section{INTRODUCTION}

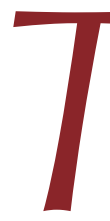

he aim of endodontic treatment is the cleansing, disinfecting and sealing of the root canal system. It has been demonstrated that a significant number of teeth have lateral canals along the root (1). These canals create a possible pathway for bacterial leakage. Wein demonstrated the importance of lateral canals in failure of endodontic treatments (2). Zolty et al also confirmed this result in another study (3). The smear layer contains organic and inorganic debris and is formed following instrumentation of the root canal, covers the dentin, and blocks the dentinal tubules orifices (3). The smear layer may potentially block the orifice of lateral canals and prevent the penetration of root canal filling material into the lateral canals. Further, the presence of the smear layer may prevent endodontic filling materials from penetrating into the dentinal tubules and sticking to the canal walls $(4,5)$. Goldberg et al indicated that the use of 15\% EDTA for removing the smear layer increased the filling rate of the lateral canals compared with $5 \% \mathrm{NaOCl}$ and distilled water (6).

The type and composition of filling materials can potentially influence the sealing of the lateral canals. Recently, calcium silicate-based sealers have been introduced in order to achieve the appropriate biological and sealing characteristics similar to those of MTA, one of which is MTAFillapex (Angelus Industria Produtos odontologicos S/A-Brasil), which is presented in the paste/ paste system which has a high sealing ability $(7,8)$. Studies have shown its excellent biocompatibility, bioactivity, and osteoconductivity (9-11).

Endoseal MTA (Maruchi-Korea) is regarded as another calcium silicate based sealer, which can be injected into the canal with a syringe. It is expected to have favorable biological and physi- cal properties such as other MTA-derived materials (12-14).

Sure-Seal Root (Sure Dent Corporation-Korea) is another calcium silicate based sealer which is typically premixed and ready to fill the canals, showing excellent physical properties and lack of shrinkage during the setting process, and as claimed by the manufacturer, demonstrates the hydrophilic properties, biocompatibility, formation of hydroxyapatite, ideal setting and working time (15).

Since the ability of new calcium silicate based sealers such as MTA Fillapex, Endoseal MTA and Sure-Seal Root has not been studied in the filling of lateral canals, the present study aimed to compare the ability to fill the lateral canals with these three sealers and an epoxy resin sealer (AH26) (Dentsply Detrey GmbH-Germany) in the presence and absence of smear layer. $\square$

\section{MATERIALS AND METHODS}

\section{Celection of samples and preparation of $\checkmark$ artificial lateral canals}

Single-rooted human teeth were used in the present study. Teeth with decay, restoration, or filled canal, severe root curvature, open apex or fracture were excluded. Finally, 80 teeth were selected for the study. The crown of each tooth was cut off by a diamond disc (Jota-Switzerland) in order to reach a standard length of $15 \mathrm{~mm}$. Six lateral canals - three in mesial and three in the distal aspect of root - were prepared at 3, 6 and $9 \mathrm{~mm}$ from apex using a size $15.2 \%$ taper engine reamer (Mani, INC-Japan). Patency of the lateral canals was confirmed by \#10k-file (ManiJapan).

\section{Preparation and obturation of root canals}

One operator prepared all canals. The working length was determined by the \#10 k-file. The 
canals were prepared by using the RaCe Rotary (FKG-Lachaux-de fonds-Switzerland) System and Crown Down Technique, The RaCe files $\# 35$, 6\% taper \#30 and \#25, 4\% taper were used to reach the working length.

After using each file, the canals were washed with $1 \mathrm{~mL}$ of $5.25 \% \mathrm{NaOCl}$. Then, teeth were randomly divided into two groups, A and $\mathrm{B}$. In group $A(n=40)$, the smear layer was removed using $5 \mathrm{~mL}$ of $17 \%$ EDTA (Dentonics-Bristol, UK) for one minute, followed by $5 \mathrm{~mL}$ of $5.25 \%$ $\mathrm{NaOCl}$, and finally canals were rinsed with $5 \mathrm{~mL}$ of saline. In group $B(n=40)$, the smear layer was not removed., the final irrigation was performed with saline and finally, canals were dried up with the paper points.

Samples in each group were divided into the following four subgroups, according to the sealer used to fill the canals: A1, B1 (MTA Fillapex); A2, B2 (Endoseal MTA); A3, B3 (Sure-Seal Root); and $\mathrm{A} 4, \mathrm{~B} 4$ (AH26).

All prepared canals were filled up by warm vertical compaction technique as follows: a Master Cone (MC) with tugback at $1 \mathrm{~mm}$ short of the working length was selected. After applying sealer to the canal walls, using a size 30 Lentulo spiral (Mani-Japan), the MC was placed in the canal. A heat plugger \#40 with a 3\% taper associated with the Beefill 2 in 1 (VDW-Germany) system was activated at $200^{\circ} \mathrm{C}$ and applied with apical pressure up to $5 \mathrm{~mm}$ of the apical foramen. This plugger warmed the MC and separated the upper part. Machotou Hand Plugger \#1 was applied for packing gutta-percha in the apical third of canals. Backfill was performed by warming up small pieces of gutta-percha and squeezing them by a large size hand plugger (size 3) until the canals were completely filled. Following canal obturation, teeth were incubated at $37^{\circ} \mathrm{C}$ and $95 \%$ humidity for 72 hours.

\section{Clearing technique}

A modified clearing technique was used to make samples transparent $(16,17)$. First, teeth were immersed in 5\% nitric acid for five days and the solution was replaced daily. In the next step, teeth were washed under tap water for four hours and rested at $80 \%, 90 \%$ and $100 \%$ ethyl alcohol ascending concentrations for one hour. Finally, they were immersed in methyl salicylate to become transparent.

\section{Stereomicroscopic analysis}

Teeth were analyzed under stereomicroscope (Nikon-Japan) from Buccal, Lingual, Mesial and Distal aspects at $10 x$ to $75 x$ magnification. The filling rate of each lateral canal was scored as follows: score 1 - less than $50 \%$ of the length of the canal filled with sealer without the presence of gutta-percha; score 2 - the canal completely filled with sealer or $50-90 \%$ of the canal length was filled with sealer and gutta-percha; and score 3 - the canal completely filled with sealer and gutta-percha (Figure 1). Score 1 was defined as unacceptable, while scores 2 and 3 were considered acceptable (Table 1).

\section{Data analysis}

The chi-square test was used to compare not only the filling rate of lateral canals in the presence and absence of the smear layer, but also the ability to fill the lateral canals using different

TAB LE 1. Laboratory values on admission

\begin{tabular}{llll}
\hline Smear Layer & Sealer & $\begin{array}{l}\text { Acceptable } \\
(\text { scores 2, 3) }\end{array}$ & $\begin{array}{l}\text { Unacceptable } \\
(\text { score 1) }\end{array}$ \\
\hline & MTA Fillapex & $25^{\mathrm{a}}$ & 35 \\
Removal of smear & Endoseal MTA & $51^{\mathrm{b}}$ & 9 \\
layer (group A) & Sure-Seal Root & $44^{\mathrm{b}}$ & 16 \\
& AH26 & $29^{\mathrm{a}}$ & 31 \\
& MTA Fillapex & $21^{\mathrm{ab}}$ & 39 \\
Presence of smear & Endoseal MTA & $36^{\mathrm{ab}}$ & 24 \\
layer (group B) & Sure-Seal Root & $38^{\mathrm{ab}}$ & 22 \\
& AH26 & $28^{\mathrm{ab}}$ & 32 \\
\hline
\end{tabular}

*Different letters within the columns indicate statistically significant differences among groups 


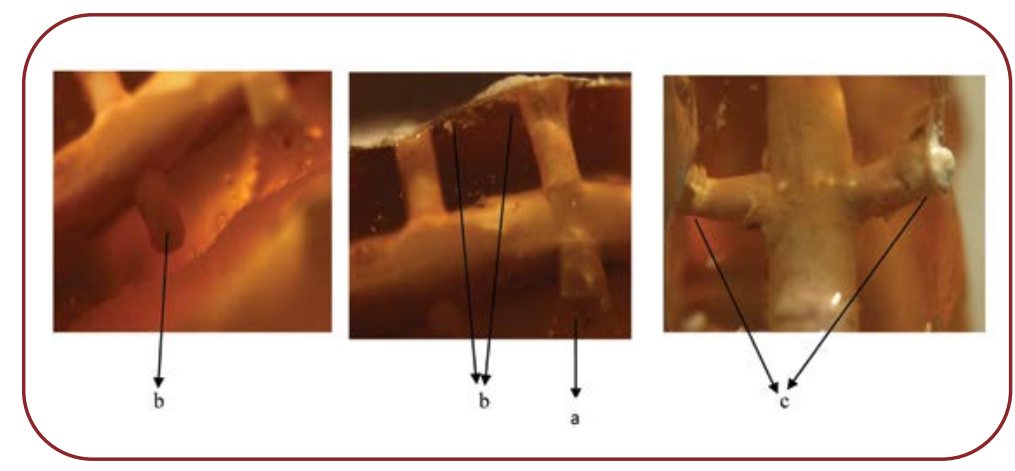

FIGURE 1. Stereomicroscopic images of the artificial lateral canals and the main canal with different filling scores (a: score 1 ; b: score 2; c: score 3)

sealers. All analyses were performed using SPSS software and $P<0.05$ was considered significance.

\section{RESULTS}

n group A, the acceptable filling rate of the lateral canals in Endoseal MTA and Sure-Seal Root groups was significantly greater than the MTA Fillapex and AH26 groups (Table 1). However, in group B, no statistically significant difference between groups was seen $(P>0.05)$.

In group $A$, in which the smear layer was removed, the filling rate of lateral canals was higher in all subgroups comparatively with subgroups of the same name in group B, although the difference was not statistically significant $(\mathrm{P}>0.05)$.

When considering the filling rate of the lateral canals with respect to the position of the canal created in the root (apical/middle/coronal third), no statistically significant difference was observed between hroups A and B and their subgroups.

\section{DISCUSSION}

L ateral canals are pathways for bacteria and their by-products to pass between the root canal and the periodontal tissue. Studies have shown that the complete cleansing and sealing of the main canal and accessory canals leads to lesion recovery $(18,19)$. To simulate clinical conditions, we formed the lateral canals using a size 15 engine reamer and a low-speed handpiece, according to the method provided by Goldberg et al (20). The results indicated that the thermoplastic gutta-percha technique in filling the lateral canals was more effective than the cold la- teral compaction technique (20). Therefore, we used the warm vertical technique to fill the canals.

This study showed that the calcium silicatebased sealers Sure-Seal Root and Endoseal MTA caused a higher filling rate of the lateral canals than the other two sealers, which was significant in the case of removing the smear layer. The finding may be explained by the higher flow of these sealers $(21,22)$. In addition, Eun-Su Lim et al demonstrated that Endoseal MTA had a clearly higher flow than the $\mathrm{AH}$ plus, a resin sealer (23). In another study, Yan Huang et al indicated that Sure-Seal Root sealer demonstrated a lower void and a high percentage of canal filling than $\mathrm{AH}$ plus resin sealer (24). The high flow rate of Calcium silicate-based sealers may be attributed to their small particles (less than $2 \mu \mathrm{m}$ ), which cause more penetrability into ramifications and lateral canals $(21,22)$.

Razavian $\mathrm{H}$ et al reported that $\mathrm{AH} 26$ sealing ability was significantly higher than that of MTA Fillapex sealer (25). In another study, Tiago Andre et al showed there was no significant difference AH26 and MTA Fillapex sealers in filling the lateral canals (26). In the present study, the filling rate of the lateral canals using $\mathrm{AH} 26$ was higher than that of MTA Fillapex, but the difference was not significant.

Another finding of the present study was that acceptable filling of lateral canals in the presence of smear layer was similar for Calcium silicatebased and resin based sealers - in other words, in the presence of smear layer even Endoseal MTA and Sure-Seal Root sealers with greater flow are unable to fill the lateral canals significantly better than $\mathrm{AH} 26$ and MTA Fillapex sealers.

In the present study, no significant difference was observed between the ability of sealers to fill the lateral canals with respect to their position in the canal, which was in accordance with the result obtained by Souza et al, who found no difference in the ability of six types of sealers (except $\mathrm{AH} 26)$ in filling the lateral canals with respect to their position (27).

\section{CONCLUSIONS}

onsidering the limitations of this study, the results indicated that: 1) Removing the smear layer increases the successful filling of the 
lateral canals by calcium silicate-based sealers, endoseal MTA and Sure-Seal Root, and 2) In the case of removing the smear layer, calcium silicate-based sealers Sure-Seal Root and Endoseal MTA are more successful in filling the lateral canals than the $\mathrm{AH} 26$ and MTA Fillapax sealers.
Conflicts of interests: none declared

Financial support: Vice-Chancellor of Tabriz

University of Medical Sciences.

Acknowledgments: The authors would like to thank the Vice-Chancellor of Tabriz University of Medical Sciences for the cooperation in this study.

\section{$\mathbf{R}_{\text {EFERENCES }}$}

1. De Deus Q, Horizonte B. Frequency, location, and direction of the lateral, secondary, and accessory canals. Journal of Endodontics 1975;1:361-366.

2. Weine F. The enigma of the lateral canal. Dental Clinics of North America 1984;28:833-852.

3. Violich D, Chandler N. The smear layer in endodontics-a review. International Endodontic Journal 2010;43:2-15.

4. Okşan T, Aktener B, Şen B, Tezel H. The penetration of root canal sealers into dentinai tubules. A scanning electron microscopic study. International Endodontic Journal 1993;26:301-305.

5. Saleh I, Ruyter I, Haapasalo M, Ørstavik D. Bacterial penetration along different root canal filling materials in the presence or absence of smear layer. International Endodontic Journal 2008;41:32-40.

6. Goldberg F, Massone JE, Spielberg C. Effect of irrigation solutions on the filling of lateral root canals. Dental Traumatology 1986;2:65-66.

7. Assmann E, Scarparo RK, Böttcher DE, Grecca FS. Dentin bond strength of two mineral trioxide aggregate-based and one epoxy resin-based sealers. Journal of Endodontics 2012;38:219-221.

8. Camilleri J. Evaluation of selected properties of mineral trioxide aggregate sealer cement. Journal of Endodontics 2009;35:1412-1417.

9. Ghasemi N. Antimicrobial effects of Apexit Plus, Epiphany, MTA Fillapex and Dorifill sealers on Enterococcus faecalis at different time intervals: Tabriz University; 2015.

10. Reyhani M, Ghasemi N, Rahimi S, et al. Apical microleakage of AH Plus and MTA Fillapex (R) sealers in association with immediate and delayed post space preparation: a bacterial leakage study. Minerva Stomatol 2015;64:129-134.

11. Reyhani MF, Ghasemi N, Rahimi S, et al. Push-out bond strength of Dorifill, Epiphany and MTA-Fillapex sealers to root canal dentin with and without smear layer. Iranian Endodontic Journal 2014;9:246.

12. Lee S-J, Chung J, Na H-S, et al. Characteristics of novel root-end filling material using epoxy resin and Portland cement.

Clinical Oral Investigations 2013;17:1009-1015.

13. Viana Viola $\mathbf{N}$, Maria Guerreiro-Tanomaru J, Ferreira da Silva G, et al. Biocompatibility of an experimental MTA sealer implanted in the rat subcutaneous: quantitative and immunohistochemical evaluation.

Journal of Biomedical Materials Research Part B: Applied Biomaterials 2012;100:1773-1781.

14. Willershausen I, Wolf T, Kasaj A, et al. Influence of a bioceramic root end material and mineral trioxide aggregates on fibroblasts and osteoblasts. Archives of Oral Biology 2013;58:1232-1237.

15. Kim I-Y, Seong B-R, Lee M-H. Triple matrix capsulation having visible effects and stabilizing functions. J. of Korean Oil Chemists' Soc 2015;32:326-329.

16. Kartal N, Yanıkoğlu FÇ. Root canal morphology of mandibular incisors. Journal of Endodontics 1992;18:562-564.

17. Rahimi S, Shahi S, Lotfi M, et al. Root canal configuration and the prevalence of C-shaped canals in mandibular second molars in an Iranian population. Journal of Oral Science 2008;50:9-13.

18. Schilder H. Cleaning and shaping the root canal.
Dent Clin North Am 1974;18:269-296.

19. Zolty G. The prevalence and significance of sealing accessory and lateral canals: a literature review. SADI 2001;56:417-424.

20. Goldberg F, Artaza LP, De Silvio A. Effectiveness of different obturation techniques in the filling of simulated lateral canals. Journal of Endodontics 2001;27:362-364.

21. Ersahan S, Aydin C. Dislocation resistance of iRoot $\mathrm{SP}$, a calcium silicate-based sealer, from radicular dentine. Journal of Endodontics 2010;36:2000-2002.

22. Nagas E, Uyanik MO, Eymirli A, et al. Dentin moisture conditions affect the adhesion of root canal sealers. Journal of Endodontics 2012;38:240-244.

23. Lim E-S, Park Y-B, Kwon Y-S, et al. Physical properties and biocompatibility of an injectable calcium-silicate-based root canal sealer: in vitro and in vivo study. BMC Oral Health 2015;15:129.

24. Huang Y, Celikten B, de Faria Vasconcelos $\mathrm{K}$, et al. Micro-CT and nano-CT analysis of filling quality of three different endodontic sealers. Dentomaxillofacial Radiology 2017;46:2017-0223.

25. Razavian H, Barekatain B, Shadmehr E, et al. Bacterial leakage in root canals filled with resin-based and mineral trioxide aggregate-based sealers. Dental Research Journal 2014;11:599.

26. Fontoura de Melo TA, Pereira Nunes D, de Carvalho Modaffar Al-Alam F, et al. Filling analysis of artificial lateral canals after main canal obturation through three different endodontic sealers. RSBO Revista Sul-Brasileira de Odontologia 2014;11:369-374. 